\title{
USO DE FERRAMENTAS DE GESTÃO PARA DEFINIÇÃO DE ESTRATÉGIAS MUNICIPAIS DE REDUÇÃO DE RISCOS DE DESASTRES
}

Daniel Pericles de Oliveira Bland de Freitas International Paper, Mogi Guaçu, São Paulo, danielbland@poli.ufrj.br

Marcos Barreto Mendonça Escola Politécnica - Universidade Federal do Rio de Janeiro, Rio de Janeiro, mbm@poli.ufrj.br

Gilberto Olympio Mota Fialho

Escola Politécnica - Universidade Federal do Rio de Janeiro, Rio de Janeiro, fiaIho@poli.ufrj.br

\section{RESUMO}

É comum enfrentar dificuldades na execução de ações de redução de riscos de desastres na gestão municipal. Para atenuar essa situação, o uso de métodos de gestão se torna atrativo para, principalmente, identificação de entraves e buscar de soluções. Com esse foco, as ferramentas "Modelo Canvas", "Brainstorming", Matriz GUT e Matriz de Preferência podem ser usadas para mapear as ações da Defesa Civil, o nível de prioridade de cada problema e as soluções mais indicadas a partir da participação interinstitucional dos órgãos envolvidos na gestão municipal de riscos de desastres. $\mathrm{O}$ presente trabalho apresenta um estudo de caso do Município de Tanguá, situado na região metropolitana do Rio de Janeiro, tendo como foco os riscos associados a inundações e deslizamentos. São descritas as diversas etapas para a aplicação do método em 2018 e as ferramentas empregadas. Os resultados mostram que os problemas a serem priorizados no município foram "Fiscalização do uso e ocupação do solo", "Recursos financeiros para ações de redução de riscos" e "Interação dos órgãos públicos entrei si e com a população". Por sua vez, as soluções mais indicadas foram "Realizar mapeamento de riscos", "Aumentar a eficiência de procedimentos de loteamentos", "Buscar ações contínuas de captação de recursos" e "Capacitação e educação da sociedade sobre riscos e sua gestão".

Palavras-chave: Defesa Civil. Redução de Riscos de Desastres. Gerenciamento de Projetos. Tanguá. 


\section{DIAGNOSIS OF MUNICIPAL DISASTER RISK REDUCTION STRATEGIES THROUGH PROJECT MANAGEMENT TOOLS}

\section{ABSTRACT}

It is common to face difficulties in the execution of actions of disaster risk reduction in town management. To mitigate this situation, the use of management methods become attractive for identify obstacles and search for solutions. With this focus, the tools called "Canvas Model", "Brainstorming", GUT Matrix and Matrix of Preference can be used to map Civil Defense actions, the priority level of each problem and the most indicated solutions based on the participation of several departments involved in a town disaster risk management. The present work presents a case of the city of Tangua, located in the metropolitan region of Rio de Janeiro, focusing on the risks associated with floods and landslides. The various steps for applying the method in 2018 and the tools employed are described. The results show that the problems to be prioritized are "Supervision of land use and occupation", "Financial resources for risk reduction actions" and "Interaction of public agencies between and with the population". In turn, the most indicated solutions are "Carry out risk mapping", "Increase the efficiency of allotment procedures", "Search for continuous fundraising actions" and "Train and educate the society about risks and their management".

Keywords: Civil Defense. Disaster Risk Reduction. Project Management. Tangua

\section{INTRODUÇÃO}

A gestão de riscos pode ser compreendida por ações específicas que visem controlar e reduzir riscos com foco em mitigar o potencial de dano caso algum evento relacionado à ameaça respectiva ocorra (UNISDR, 2009).

Os desastres associados a deslizamentos de terra e inundações vêm aumentando em magnitude, frequência e território envolvido (CEPED/UFSC, 2013), evidenciando que a diretriz de gestão de riscos que vem sendo adotada há décadas, baseada em ações estruturais (obras de engenharia), tem sido deficiente. Diante desse quadro, deve-se investir em ações não estruturais para a redução das diversas dimensões de vulnerabilidade da sociedade frente aos riscos de desastres. Entre as ações não estruturais, destacam-se as ações de treinamento e capacitação dos agentes públicos para a gestão dos riscos (KOBIYAMA et al., 2006). Percebe-se uma demanda de capacitação desses agentes públicos não só quanto ao mapeamento de perigo e de risco e definição das ações de redução de riscos, mas também para a identificação de problemas e soluções para se alcançar uma gestão de riscos mais eficiente.

Diante disso, apresenta-se, neste trabalho, um método para atender a esta demanda de se buscar estratégias para uma gestão de riscos mais eficiente através do uso de ferramentas correntemente usadas em gestão de projetos, tendo como estudo de caso o município de Tanguá, situado na região metropolitana do estado do Rio de Janei- 
ro.

O município de Tanguá, com 30.732 habitantes em 2010 (IBGE, 2010), apresenta, historicamente, uma grande incidência de danos associados a inundações, com seis ocorrências entre 1991 e 2012. Nesse período, pode-se destacar o caso de 2010 que resultou em 248 desabrigados e 22.800 pessoas afetadas (BRASIL, 2011), o que representou à época, aproximadamente, $75 \%$ de sua população.

O objetivo deste trabalho é demonstrar a aplicação do conjunto de ferramentas de gestão selecionadas especificamente para a gestão municipal de riscos de desastres de forma a evidenciar os principais problemas e as respectivas soluções estruturais e/ou não estruturais, considerando as experiências de profissionais de diversos órgãos governamentais e da sociedade civil.

\section{METODOLOGIA}

A aplicação das ferramentas propostas em Tanguá ocorreu em junho 2018, sendo dividida em três etapas, cada uma relacionada a aplicação de um ferramenta de gestão - Modelo Canvas, Brainstorming /Matriz GUT e Brainstorming/Matriz de Preferência - as quais foram coordenadas pelos autores do presente trabalho (FREITAS, 2018).

A primeira etapa consistiu na aplicação do Modelo Canvas adaptado para a atuação da Defesa Civil, que visa ter uma visão sistêmica da coordenadoria local através do levantamento dos itens indicados na Tabela 1. Buscou-se mapear funções e conexões mais importantes para o funcionamento efetivo da Coordenadoria de Defesa Civil Municipal através de uma reunião com quatro profissionais que compõem a equipe, incluindo o coordenador (Figura 1). Uma das funções da ferramenta é poder identificar de forma objetiva os parceiros-chave para, em seguida, convidá-los a participarem das dinâmicas envolvidas nas etapas posteriores. Para tal, requisitos foram determinados para avaliar de forma mais refinada quem podem ser os participantes das próximas oficinas, conforme segue (FREITAS, 2018):

Dependência da Defesa Civil para atuar mais eficientemente na redução de riscos;

Cobrir todas as esferas da Defesa Civil no âmbito de redução de desastres (prevenção, gestão financeira, capacitação técnica, preparação e resposta aos desastres);

Quantidade de vezes que o parceiro foi citado pelos participantes da atividade de preenchimento do modelo Canvas adaptado;

Limitar a quantidade de participantes em até 14 pessoas para cada etapa. 
Tabela 1 - Conceito de cada item a ser preenchido no Modelo Canvas (FREITAS, 2018)

\begin{tabular}{|l|l|c|}
\hline \multicolumn{1}{|c|}{ Item } & \multicolumn{1}{|c|}{ Conceito } & Ordem de definição \\
\hline Parcerias-chave & $\begin{array}{l}\text { Entender quais são os órgãos e entes conectados com } \\
\text { a Defesa Civil para que ela desempenhe sua função }\end{array}$ & 1 \\
\hline Ativididades-chave & $\begin{array}{l}\text { Quais são os ferramentais necessários para o pleno } \\
\text { funcionamento da Defesa Civil }\end{array}$ & 2 \\
\hline Recursos chave & $\begin{array}{l}\text { Recursos fisicos necessários para gerar resultados } \\
\text { mais assertivos para os segmentos da população }\end{array}$ & 3 \\
\hline Segmentos da população & $\begin{array}{l}\text { Quem são e onde estão os segmentos mais atendidos } \\
\text { pela Defesa Civil e seu perfil socioeconômico }\end{array}$ & 4 \\
\hline Relação com os clientes & Como passam informações gerais para a população & 5 \\
\hline Canais & $\begin{array}{l}\text { Como a Defesa Civil fornece o seu trabalho e como a } \\
\text { população se comunica com o órgão }\end{array}$ & 6 \\
\hline Estrutura de custos & $\begin{array}{l}\text { Custos mais importantes para o pleno funcionamento } \\
\text { da Defesa Civil }\end{array}$ & 8 \\
\hline Fontes de Receita & $\begin{array}{l}\text { Determinar todos os financiadores da atuação da } \\
\text { Defesa Civil }\end{array}$ & 9 \\
\hline Atuações da Defesa Civil & $\begin{array}{l}\text { Luais or problemas são solucionados e quais as } \\
\text { necessidades da população }\end{array}$ & 7 \\
\hline
\end{tabular}

A segunda etapa consistiu na realização de um Brainstorming e da aplicação da Matriz GUT, a partir de uma dinâmica com dez integrantes de diferentes secretarias da prefeitura e representantes da sociedade civil, os quais foram selecionados na etapa anterior. Após uma breve apresentação oral sobre gestão de desastres geo-hidrológicos, realizada por um especialista, o Brainstorming permitiu que fossem enumerados, por todos os integrantes da reunião, os problemas da gestão de riscos municipal. Posteriormente, a Matriz GUT foi usada para priorizar os problemas com base nos elementos "Gravidade", Urgência" e "Tendência" para cada problema, sendo cada elemento pontuado de 1 a 5 como mostra a Tabela 2 (BEZERRA et al., 2012). Com a pontuação de cada elemento, tem-se o produto $\mathrm{G} \times \mathrm{U} \times \mathrm{T}$ e, assim, a priorização dos problemas. 
Tabela 2 - Significado da pontuação dos problemas (DAYCHOUM, 2016)

\begin{tabular}{|c|c|c|c|c|}
\hline \multirow[b]{2}{*}{ Pontos } & $\mathrm{G}$ & $\mathrm{U}$ & $\mathrm{T}$ & \multirow[b]{2}{*}{ GxUxT } \\
\hline & $\begin{array}{c}\text { Gravidade } \\
\text { Consequências se nada for } \\
\text { feito }\end{array}$ & $\begin{array}{c}\text { Urgência } \\
\text { Prazo para tomada de decisão }\end{array}$ & $\begin{array}{c}\text { Tendência } \\
\text { Proporção do problema no } \\
\text { futuro }\end{array}$ & \\
\hline 5 & $\begin{array}{l}\text { Os prejuízos ou dificuldades } \\
\text { são extremamente graves }\end{array}$ & $\begin{array}{c}\text { É necessária uma ação } \\
\text { imediata }\end{array}$ & $\begin{array}{c}\text { Se nada for feito, o } \\
\text { agravamento da situação será } \\
\text { imediato }\end{array}$ & $5 \times 5 \times 5=125$ \\
\hline 4 & Muito graves & Com alguma urgência & Vai piorar em curto prazo & $4 \times 4 \times 4=64$ \\
\hline 3 & Graves & O mais cedo possível & Vai piorar em médio prazo & $3 \times 3 \times 3=27$ \\
\hline 2 & Pouco graves & Pode esperar um pouco & Vai piorar em longo prazo & $2 \times 2 \times 2=8$ \\
\hline 1 & sem gravidade & Não tem pressa & $\begin{array}{l}\text { Não vai piorar e pode até } \\
\text { melhorar }\end{array}$ & $1 \times 1 \times 1=1$ \\
\hline
\end{tabular}

A última dinâmica, que consistiu na terceira etapa, tratou de uma nova aplicação de Brainstorming, agora enumerando as soluções para cada um dos problemas prioritários. Logo após, a Matriz de Preferência foi usada por cada integrante, a partir da qual se pode identificar as soluções preferidas comparando-as uma a uma.

O envolvimento de diversos atores e a análise conjunta dos resultados confere ao método uma característica local, participativa, interdisciplinar e interinstitucional.

\section{RESULTADOS E DISCUSSÃO}

Com o Modelo Canvas foi possível determinar os parceiros-chave do órgão que deveriam participar das dinâmicas posteriores para a aplicação do método.

O primeiro Brainstorming identificou quinze problemas da gestão de risco em Tanguá. Cada problema foi pontuado, individualmente, segundo sua gravidade, urgência e tendência (Matriz GUT). Os quinze problemas foram agrupados em seis conjuntos, sendo eles: (1.1) Fiscalização do uso e ocupação do solo; (1.2) Recursos financeiros para ações de redução de riscos; (1.3) Interação dos órgãos públicos entre si e com a população; (1.4) Recursos financeiros para operação da Defesa Civil; (1.5) Gestão participativa; e (1.6) Recursos humanos. Para esses seis conjuntos foi elaborado um Diagrama de Pareto, que apresenta o peso que um conjunto de problemas apresenta frente a todos os outros determinando a SUS respectiva relevância (Figura 1). Os três principais conjuntos de problemas foram: (1.1) "Fiscalização do uso e ocupação do solo"; (1.2) "Recursos financeiros para ações de redução de riscos"; e (1.3) "Interação dos órgãos públicos entre si e com a população". 
Figura 1 - Diagrama de Pareto dos conjuntos de problemas

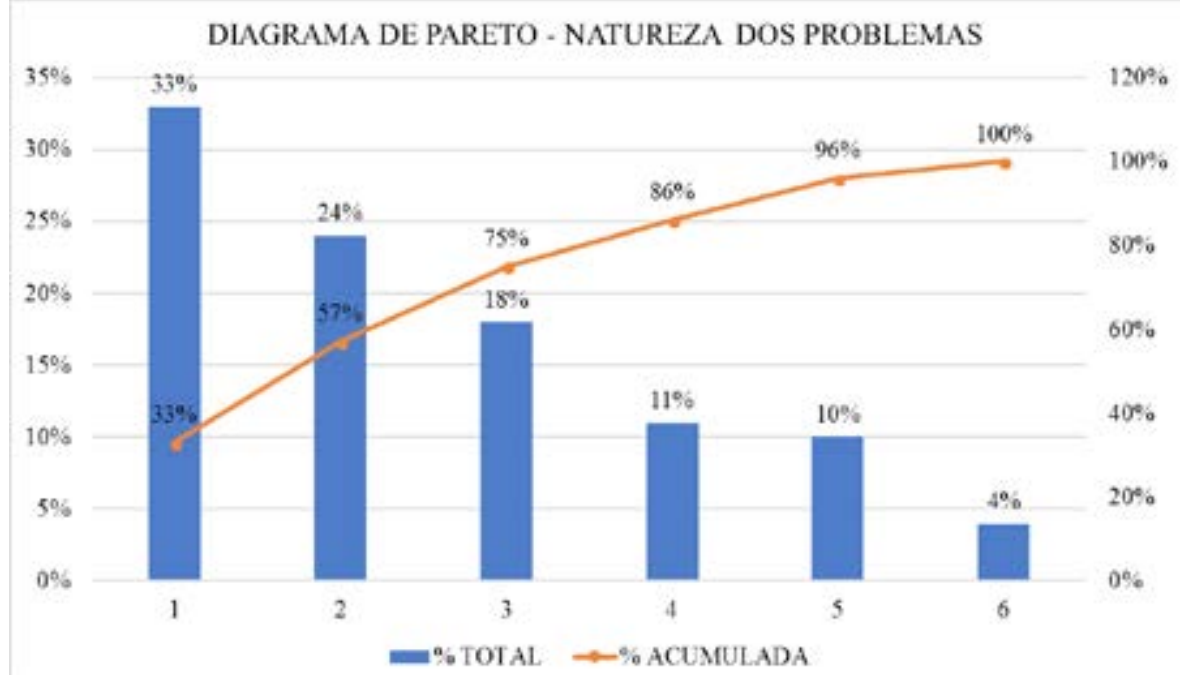

Fonte: FREITAS (2018)

Na terceira etapa buscaram-se as soluções para cada um dos três conjuntos de problemas prioritários. Após o Brainstorming para a indicação de soluções para os conjuntos de problemas por parte de cada um dos participantes, foi elaborada uma lista das soluções propostas. Através da Matriz de Preferência, os participantes indicaram as soluções que julgaram mais adequadas comparando uma a uma. As Figuras 2, 3 e 4 apresentam as soluções para os dois principais conjuntos de problema: (2.1) "Fiscalização do uso e ocupação do solo"; (2.2) "Recursos financeiros para ações de redução de riscos"; e (2.3) "Interação dos órgãos públicos entre si e com a população".

Na busca da solução mais adequada para o problema (1.1) "Fiscalização do uso e ocupação do solo", a compilação dos resultados de todos os participantes (Figura 2) evidencia que (3.1) "Fazer mapeamentos de risco e hierarquizar as áreas de risco" é a preferida com 53 indicações, seguida de (3.2) "Melhoria dos procedimentos da análise técnica de loteamentos em geral" (52 indicações). 
Figura 2 - Resultado da Matriz de Preferência para o conjunto de problemas "Fiscalização do uso e ocupação do solo" - Frequência das indicações de preferência FREQUENCIA TOTAL - SOLUÇÃO 1

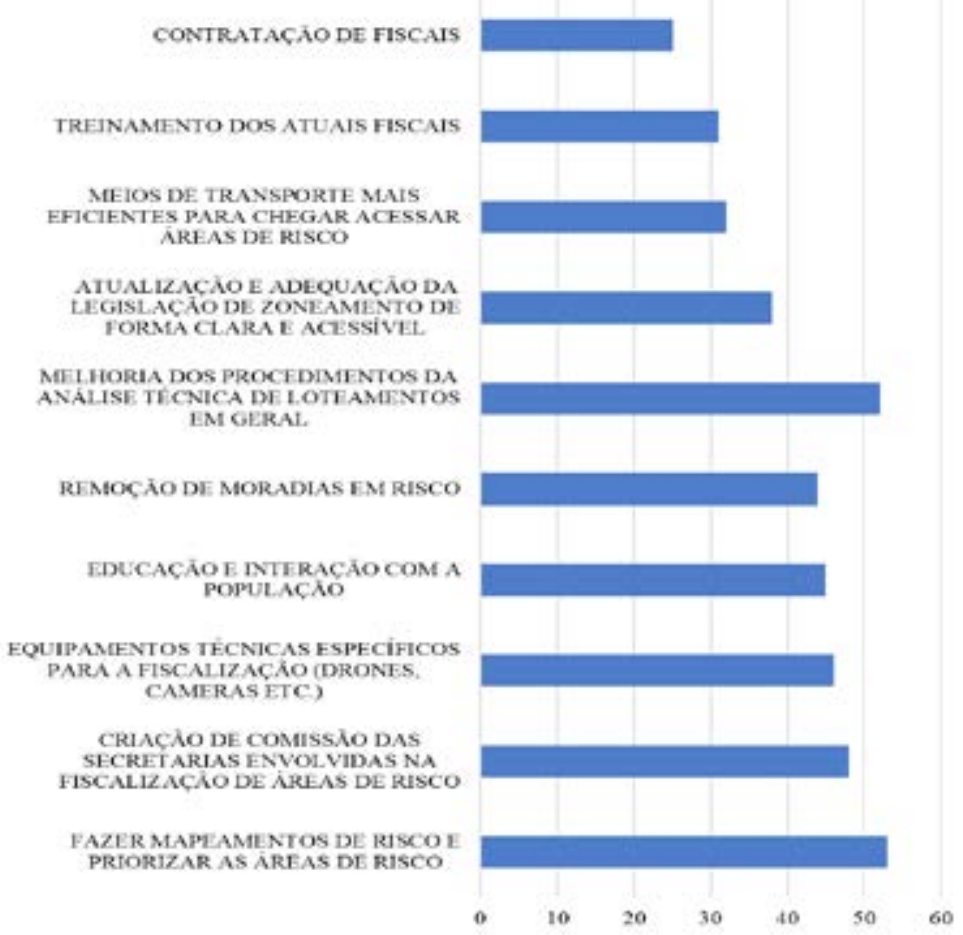

Fonte: FREITAS (2018)

O segundo conjunto de problemas avaliado é (2.2) "Recursos financeiros para ações de redução de riscos". De acordo com o levantamento de resultados, é possível observar que a opção mais escolhida pelos integrantes foi (4.1) "Ação conjunta entre secretarias para captação de recursos", com 36 votos. Vale ressaltar que (4.2) "Aumentar convênios tipo Minha Casa Minha Vida para populações de áreas de risco" e (4.3) "Capacitar pessoal da prefeitura para elaborar projetos de redução de risco para facilitar a captação de recursos federais" aparecem com 33 e 31 votos, respectivamente e devem ser consideradas como possíveis soluções para o dado problema (Figura 3). 
Figura 3 - Soluções da Matriz de Preferência para o conjunto de problemas "Recursos financeiros para ações de redução de riscos" - Frequência das indicações de preferência.

$$
\text { FREQUENCIA TOTAL - SOLUÇAO } 2
$$

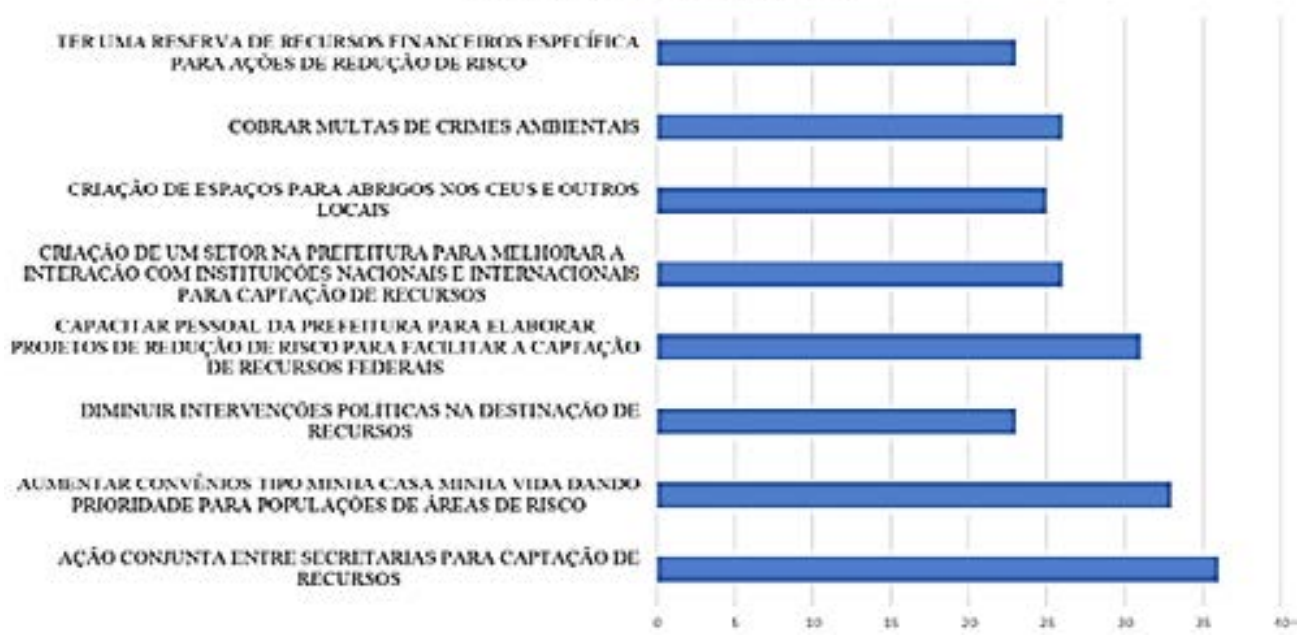

Fonte: FREITAS (2018)

O último conjunto de problemas a ser avaliado é (1.3) "Integração dos órgãos públicos entre si e com a população". A opção de solução (5.1) "Ter uma defesa civil com maior poder de decisão nas ações de redução de risco" foi a que mais se destacou, com 58 indicações. É importante observar que soluções relacionadas a medidas educacionais e de treinamento receberam entre 40 e 50 votos, variando da segunda colocação até a sétima (Figura 4). 
Figura 4 - Soluções da Matriz de Preferência para o conjunto de problemas "Comunicação dos órgãos públicos entre si e com a população"- - Frequência das indicações de preferência.

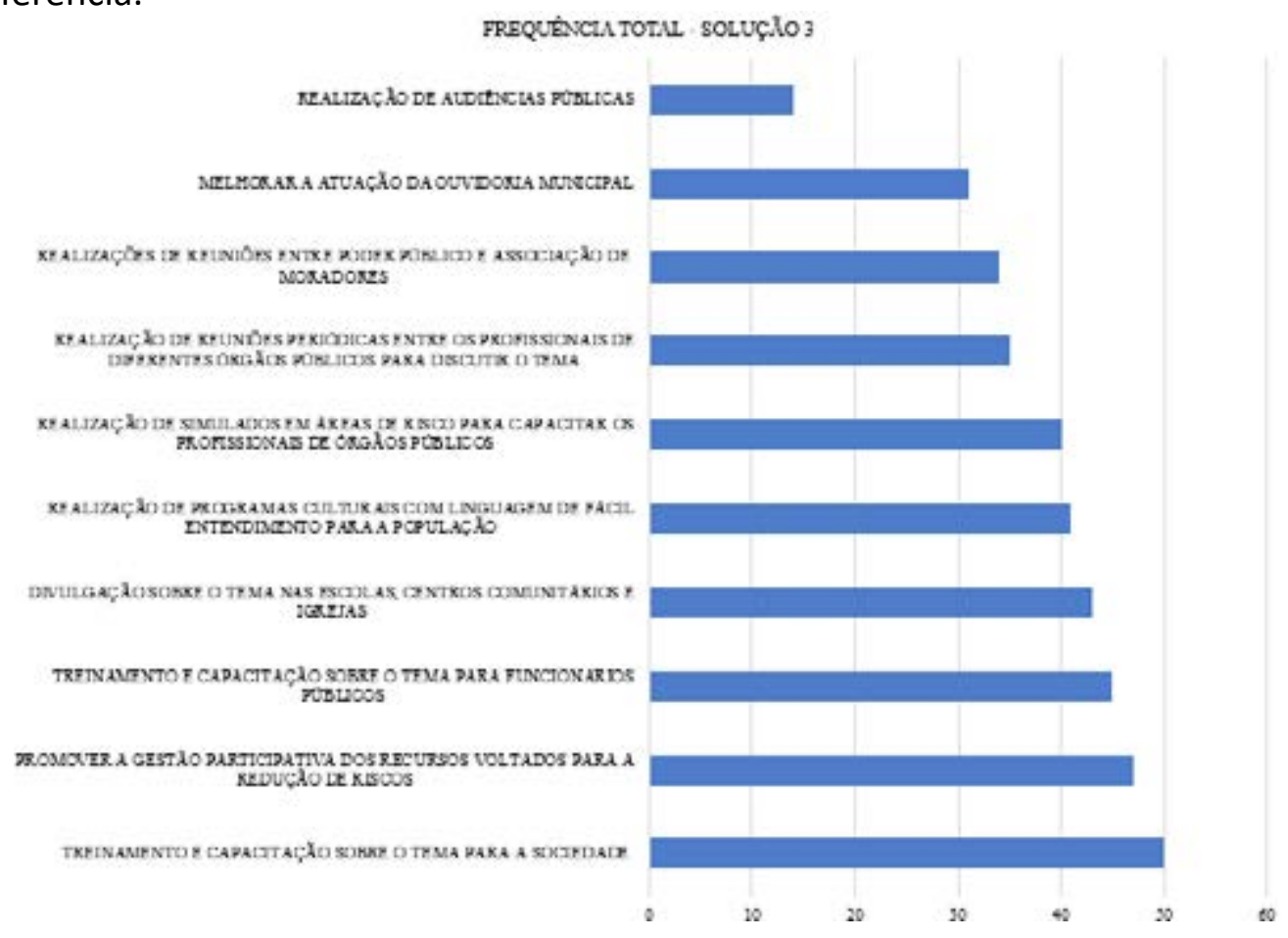

Fonte: FREITAS (2018)

\section{CONCLUSÃO}

Diante da complexidade envolvida na gestão de risco de desastres, a aplicação do conjunto de ferramentas de gestão formado pelo Modelo Canvas, Brainstorming, Matriz GUT e Matriz de Preferência para identificar problemas e soluções prioritários, conforme proposto no presente trabalho, se mostrou eficiente. A aplicação das ferramentas de gestão também se mostrou flexível de acordo com sua necessidade. Após essa primeira aplicação, essas ferramentas podem ser usadas tendo como foco um tópico específico da gestão de risco de desastres, significando um aprofundamento do estudo. O emprego do método se mostrou simples e rápido. O envolvimento de diversos atores na aplicação das ferramentas e a análise conjunta dos resultados conferiu ao mesmo uma característica local, participativa, interdisciplinar e interinstitucional ao método.

A aplicação das ferramentas no Município de Tanguá indicaram que os principais problemas relacionados a gestão de riscos de desastres foram "Fiscalização do uso e ocupação do solo"; "Recursos financeiros para ações de redução de riscos"; e "Interação dos órgãos públicos entre si e com a população". As soluções indicadas foram diversas, destacando-se "Fiscalização do uso e ocupação do solo"; "Ação conjunta entre secretarias para captação de recursos"; e "Ter uma defesa civil com maior poder de decisão nas ações de redução de risco". Os resultados apresentados no presente trabalho, referentes 
ao Município de Tanguá, são parciais, sendo parte de um relatório amplo e detalhado.

O uso do método deve ser visto como uma das primeiras etapas da gestão de riscos, não prescindindo de profissionais especializados no detalhamento das ações de redução de riscos.

\section{AGRADECIMENTO}

Os autores agradecem aos profissionais da Prefeitura Municipal de Tanguá, em especial, ao Tenente Coronel Alexander Anthony Barrera, pelo apoio e participação na aplicação das ferramentas apresentadas.

\section{REFERÊNCIAS}

BEZERRA, T. et al. Aplicação das ferramentas da qualidade para diagnóstico de melhorias numa empresa de comércio de materiais. XXXII Encontro nacional de engenharia de produção - desenvolvimento sustentável e responsabilidade social: as contribuições da engenharia de produção, p. 14, 2012.

CEPED-UFSC. Atlas brasileiro de desastres naturais 1991 a 2012: volume Brasil. 2a. ed. Florianópolis: CEPED - UFSC, 2013.

DAYCHOUM, M. No 40+16 ferramentas e técnicas de gerenciamento. 6a. ed. Rio de Janeiro: BRASPORT, 2016.

BRASIL. Anuário brasileiro de desastres naturais 2011. 1a. ed. Brasília: Ministério da Integração Nacional, 2012.

FREITAS, D. Aplicação de ferramentas de gestão para a melhoria do planejamento municipal de ações de redução de riscos associados a movimentos de massa e inundações - o caso do município de Tanguá. Trabalho de Conclusão de Curso de Engenharia Civil. Rio de Janeiro: Universidade Federal do Rio de Janeiro, Escola Politécnica, Departamento de Construção Civil, 2018.

KOBIYAMA, M. et al. Prevenção de desastres naturais - conceitos básicos. 1a. ed. Curitiba: Organic Trading, 2006.

IBGE. Tanguá - Panorama. Disponível em: <https://cidades.ibge.gov.br/brasil/rj/ tangua/panorama>. Acesso em: 1 jul. 2018.

UNITED NATIONS INTERNATIONAL STRATEGY FOR DISASTER REDUCTION. 2009 unisdr terminology on disaster risk reduction. 1a. ed. Geneva: United Nations International Strategy for Disaster Reduction, 2009. 